Review

\title{
Multiple Roles of sFRP2 in Cardiac Development and Cardiovascular Disease
}

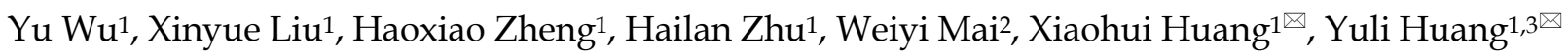 \\ 1. Department of Cardiology, Shunde hospital, Southern Medical University, Jiazi Road 1 Lunjiao Town, Shunde District, Foshan, Guangdong, 528308, China. \\ 2. Department of Cardiology, The First Affiliated Hospital of Sun Yat-sen University, 510080, Guangzhou \\ 3. The George Institute for Global Health, NSW 2042 Australia \\ $\triangle$ Corresponding authors: M.S. Xiaohui Huang, Department of Cardiology, Shunde hospital, Southern Medical University,Jiazi Road 1, Lunjiao Town, Shunde \\ District, Foshan, Guangdong, China, 523808. Tel: +86 757 22318680, Email:hxhscience@163.com; Prof. Yuli Huang, Department of Cardiology, Shunde hospital, \\ Southern Medical University,Jiazi Road 1, Lunjiao Town, Shunde District, Foshan, Guangdong, China, 523808. Tel: +86 75722318680 , \\ Email:hyuli821@smu.edu.cn; yhuang@georgeinstitute.org.au
}

(c) The author(s). This is an open access article distributed under the terms of the Creative Commons Attribution License (https://creativecommons.org/licenses/by/4.0/). See http://ivyspring.com/terms for full terms and conditions.

Received: 2019.10.05; Accepted: 2019.12.14; Published: 2020.01.14

\begin{abstract}
The Wnt signaling pathway plays important roles in organ development and disease processes. Secreted frizzled-related protein 2 (sFRP2), a vital molecule of Wnt signaling, can regulate cardiac development and cardiovascular disease. Recent studies have suggested that sFRP2 is not only an antagonist of the canonical Wnt signaling pathway, but also has a more complex relationship in myocardial fibrosis, angiogenesis, cardiac hypertrophy and cardiac regeneration. Here, we review the role of sFRP2 and Wnt signaling in cardiac development and cardiovascular disease.
\end{abstract}

Key words: Wnt Signaling, Secreted frizzled-related protein 2, Cardiac Fibrosis, Angiogenesis, Hypertrophy, Cardiac development

\section{Introduction}

The heart is one of the most essential organs for maintaining the normal operation of activities of daily living, and the development of the embryo heart and cardiovascular disease are complex processes. Exploring the regulatory mechanisms that underlie these processes will allow a deeper understanding of cardiac development and provide new therapeutic targets for cardiovascular disease.

The Wnt signaling pathway is important for maintaining the homeostasis of embryonic development, and participates in the regulation of biological processes that include cell proliferation, differentiation, apoptosis, and cell localization [1, 2].On one hand, during cardiac development, Wnt signaling is an important regulator of early cardiac stimulation. On the other hand, the Wnt signaling pathway is also involved in the regulation of cardiovascular diseases, such as cardiac fibrosis, myocardial infarction and cardiac hypertrophy.

The proteins of secreted frizzled-related protein (sFRP) family are antagonists of the Wnt signaling pathway, and sFRP2 is considered to be the most potent [3]. Recent studies showed that the role of sFRP2 is not only an antagonist for the canonical Wnt signaling pathway, but it also has a more complex relationship with the Wnt pathway in myocardial fibrosis and cardiac regeneration. This review will focus on the importance of sFRP2 in cardiac development and cardiovascular disease.

\section{Overview of Wnt Pathways and sFRP2}

The term "Wnt" is derived from a combination of two words: wingless $(w g)$, the segment polarity gene of fruit flies, and the mouse proto-oncogene int-1. In 1982, Nusse et al. first discovered a Wnt gene in the study of a viral transcriptional mechanism in the mouse mammary gland that was found to transmit growth and development signals between cells [4]. Nineteen Wnt genes have been found in mammals. Wnt proteins bind receptors on the cell membrane in an autocrine or paracrine manner to regulate target gene expression. The Wnt signaling 
pathway is widely involved in early embryo development, cell proliferation, migration, tumorigenesis, metastasis, and stem cell growth regulation [5]. Currently, three major pathways for Wnt signaling have been clearly described: the canonical Wnt/ $\beta$-catenin pathway, the non-canonical Wnt/c-jun pathway, and the Wnt/ $\mathrm{Ca}^{2+} / \mathrm{PKC}$ pathway [6].

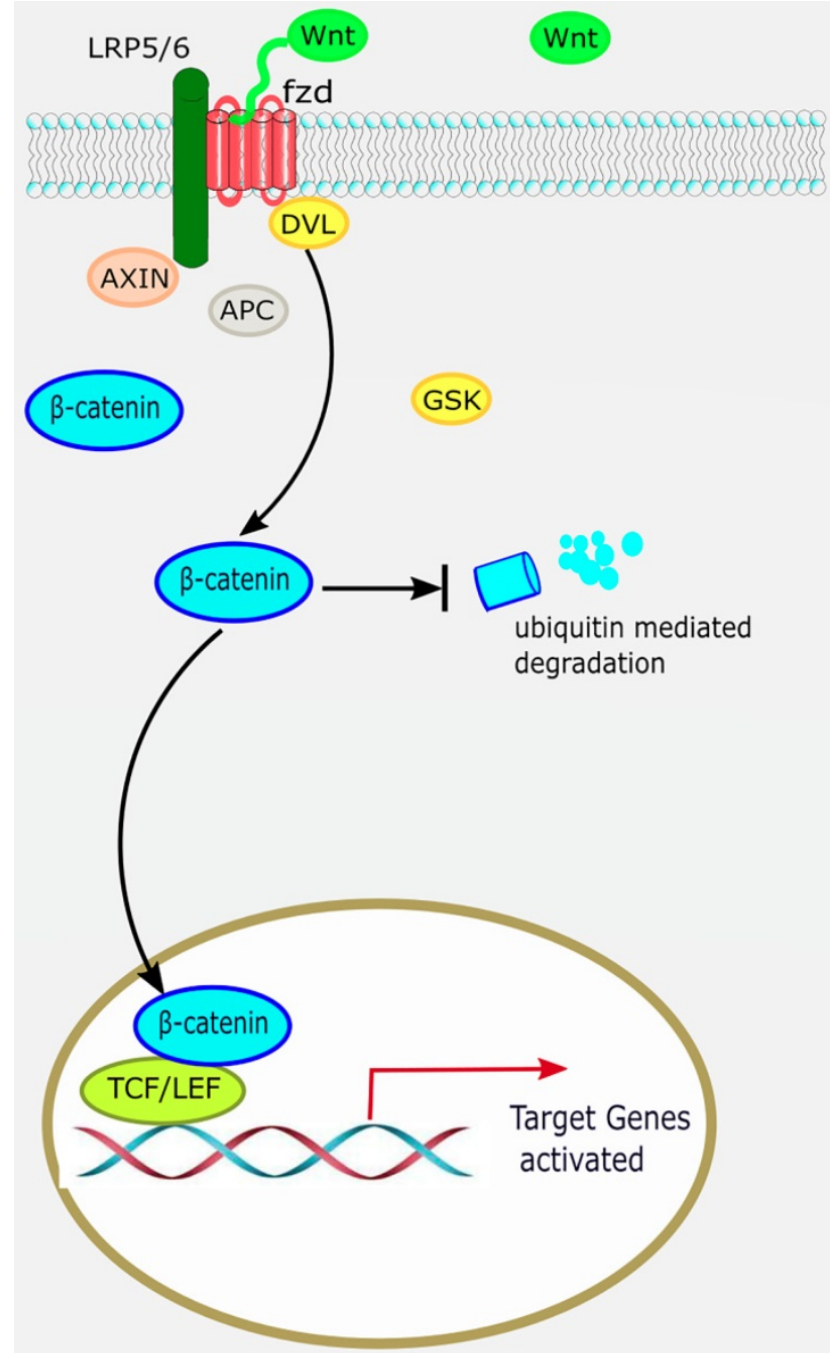

Figure 1: The canonical $W n t / \beta$-catenin pathway. When the canonical Wnt/ß-catenin pathway is "on", the receptor complex consisting of frizzled and low-density lipoprotein receptor-related protein (LRP) $5 / 6$ bind WNT, which recruits the disheveled (DVL) protein to the plasma membrane. Subsequently, several components of the $\beta$-catenin destruction complex are recruited to the membrane, where they inhibit $\beta$-catenin ubiquitination and degradation, leading to stable accumulation of $\beta$-catenin in the cytoplasm. Lymphocyte enhancement factor/ T-cell factor (LEF/TCF) then binds to $\beta$-catenin to regulate the expression of target genes downstream of the Wnt pathway.

The canonical Wnt/ $\beta$-catenin pathway is widely involved in organ development, histogenesis, regulation of the behavior and function of various precursor cells, meanwhile it is also an important regulatory factor in cardiac development. $\beta$-catenin is the most important signaling molecule of the canonical Wnt pathway, with its stability and nuclear translocation being the most important markers of canonical Wnt activation. The basic processes of the canonical Wnt/ $\beta$-catenin signaling pathway are as follows: ligand Wnt protein combines with frizzled $(F z)$ receptor to form a large complex on the cell surface with low-density lipoprotein receptor-related protein (LRP) $5 / 6$ to activate scattered protein $(D s h / D v l)$, and affect the distribution of intracellular axis protein Axin. Therefore, inhibiting the phosphorylation activity of GSK3- $\beta$ leads to stable accumulation of $\beta$-catenin in the cytoplasm [7]. $\beta$-catenin translocates to the nucleus and combines with lymphocyte enhancement factor/T-cell factor (LEF/TCF) to regulate the expression of target genes downstream of the Wnt pathway, such as $c-M y c$ and cyclin D1 (Figure 1) [8].

The sFRP family has long been considered a class of antagonists of the Wnt signaling pathway. Five members of sFRP family (Sfrp1-Sfrp5) have been identified in mammals [4-6, 9]. The sFRP family is thought to be a suppressor of Wnt signaling [10-12]. However, recent studies on the relationships between sFRPs and Wnt signaling have yielded different results. Bovolenta et al. revealed that different sFRP family proteins can both inhibit and promote Wnt signaling [13]. The sFRPs bind to Wnt through a cysteine-rich domain (CRD) or a C-terminal netrin-like domain (NTR), or bind to the frizzled receptor to form a non-functional complex that inhibits Wnt signaling. The sFRPs can also interact with each other to form dimers, or promote Wnt by either transporting Wnt proteins to frizzled or by binding to frizzled directly during signal transduction [13] (Figure 2).

Highly conserved throughout evolution, sFRP2 is widely involved in cell proliferation, apoptosis and differentiation. Its gene is located on human chromosome 4 and is about $2 \mathrm{~kb}$ in length, encoding a soluble protein of $34 \mathrm{kDa}$. The highest expression level of sFRP2 that has been found is in undifferentiated precursor adipocytes in rodents and humans [7]. Previously, sFRP2 was regarded as one of the strongest antagonist of Wnt signaling. It is interesting that, along with other sFRP family members, recent studies have raised questions about the nature of the interactions of sFPR2 in Wnt signaling. Yamamura et al. first reported that sFRP2 can activate the canonical Wnt signaling pathway in renal cell carcinoma [14]. Other studies also reported that sFRP2 can activate the canonical Wnt signaling pathway in different cell types and disease models, including lung cancer cells [15], dermal papilla cells [16], intestinal epithelial cells [17], the vertebrate optic disc [18], and cardiac fibroblast cells [19]. 


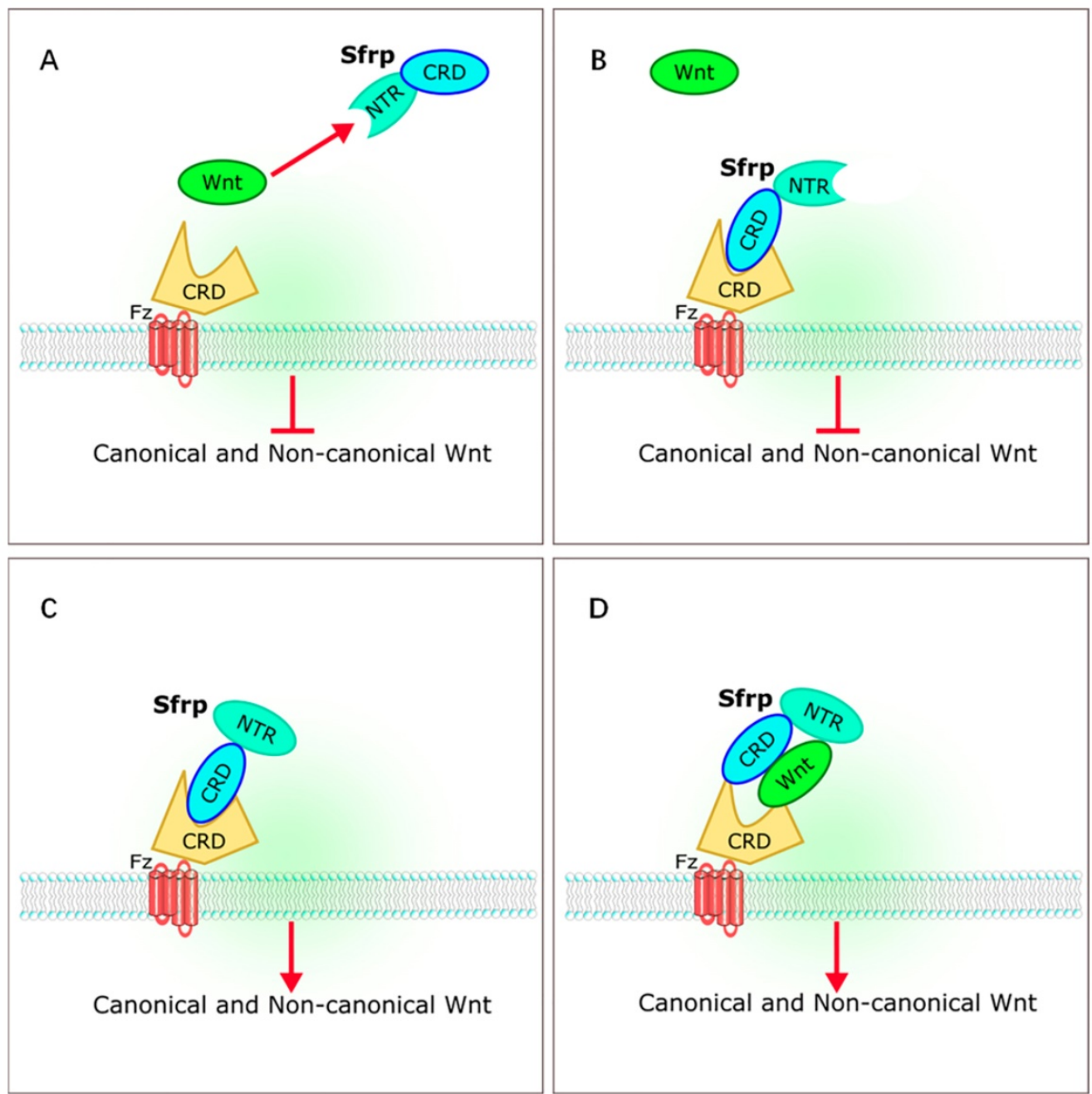

Figure 2: Interaction of sFRPs and Wnt signaling pathways. (A,B) sFRPs bind to Wnt through a cysteine-rich domain (CRD) or a C-terminal netrin-like domain (NTR), or bind to the frizzled receptor to form a non-functional complex that inhibits Wnt signaling. (C, D) sFRP interacts to form dimers, or promotes Wnt by transporting Wnt proteins to frizzled or by binding directly to frizzled during signal transduction. sFRP, secreted frizzle-related protein.

\section{The role of sFRP2 in cardiac development and cardiovascular disease}

\section{Role of sFRP2 in cardiac development}

During embryonic development of vertebrates, the heart, as the hub of the circulatory system, is the earliest functional organ to develop. Cardiac development originates from the cardiac progenitors of the lateral plate mesoderm, which begins to develop under the trigger of the induction signal [20]. The growth of the heart is extremely complicated but can be briefly summarized as four stages: 1) the establishment of the anterior plateau of the mesoderm; 2) the specialization of cardiomyocytes and endocardial progenitor cells; 3) the concentric regions of the foreguts merge to form a linear heart tube; and 4) the heart tube is cyclized and transformed into a four-chamber heart [21, 22]. Liu et al. first discovered that Wnt3a deficiency leads to the decreased expression of mesoderm markers [23].
Huelskenet al. created a knockout of $\beta$-catenin in mouse embryos and found that the mesoderm of the heart could not form, suggesting the cardiac development, particularly the mesoderm development, is dependent on activation of the canonical Wnt pathway [24]. Subsequent studies have also shown that the activation of canonical Wnt siganling pathway is essential in various stages of cardiac development, including development of cardiac neural crest cell development and outflow tract, and coronary artery formation $[25,26]$.

Nevertheless, a study of chicken and Xenopus embryos suggested that the formation of the heart depends on lower $\mathrm{Wnt} / \beta$-catenin activity [27]. How might we explain these contradictory results? Naito et al. first proposed a two-way theory of Wnt/ $\beta$-catenin signaling on cardiac developmental regulation. They found that during the differentiation of embryonic stem (ES) cells into cardiomyocytes, Wnt signaling promotes the differentiation of ES cells in the early stage, then plays a negative regulatory role in the late 
stage [28]. Ueno et al. found that, if the canonical Wnt signal is activated before the formation of the primitive gut, specialization of mesoderm cells can be stimulated and converted into a linear heart tube; in the gastrula stage, the Wnt signal must be inhibited to allow the heart to continue to develop [29]. These results led to the hypothesis that canonical Wnt $/ \beta$-catenin signaling regulates cardiac development in a bidirectional manner.

Experiments in the mouse teratoma cell line P19CL6 suggested that sFRP2 functions are dependent on the positive feedback effect of destroying autologous Wnt3a transcription, which prevents mesoderm cell specialization and inhibits P19CL6 cells from differentiating into cardiomyocytes [30]. Eisenberg et al. found that sFRP2 is expressed in the Spemann organizer and its lateral aspect in Xenopus laevis. In a study of chicken embryos, sFRP2 was not expressed in the endoderm but only in mesoderm and ectoderm derivatives [31]. During subsequent cardiac development, sFRP2 is expressed in all parts of the heart, suggesting that sFRP2 may be involved in cardiomyocyte differentiation at the cellular level, and cell migration and cardiac development throughout the development of the heart $[32,33]$.

Overall, sFRP2 exhibits spatiotemporal-specific expression during cardiac development and has bi-directional regulatory effects on the canonical Wnt signaling pathway during cardiac development. Interestingly, some studies have shown that sFRP2 can also regulate the non-canonical Wnt pathways. In mice for example, sFRP2 regulates the formation of the trunk by regulating the Wnt/PCP pathway [34]. During adult stem cell formation of Xenopus laevis intestine, sFRP2 regulates not only the canonical Wnt pathway, but also the non-canonical Wnt5a/Ror2 pathway [35].

\section{Role of sFRP2 in cardiac fibrosis}

Wnt activity is normally low in the adult organism, and it is generally believed that it is reactivated under pathological conditions. It was reported that Wnt signaling is activated shortly after myocardial infarction and is significantly associated with cardiac remodeling and fibrosis [36-38]. Duan et al. found that the canonical Wnt/ $\beta$-catenin pathway was activated after acute ischemic myocardial injury in mice. Wnt1, a canonical Wnt, was upregulated by 8 -fold in the heart within 48 hours, and cardiac fibroblasts were induced to proliferate and express profibrotic genes, eventually leading to heart repair and fibrosis [39]. After inhibiting Wnt signaling in epicardial and cardiac fibroblasts, collagen deposition and loose granulation tissue at the heart lesion area were severely reduced, and cardiac function deteriorated significantly [39]. Similarly, Lal et al. found that, in hamsters with ischemic heart cardiomyopathy, activation of the canonical Wnt pathway via inhibition of GSK3- $\beta$ promoted cardiac fibrosis by upregulating the proliferation of cardiac fibroblasts [40].

It has been reported that sFRP2 can promote the proliferation of cardiac fibroblasts by activating the Wnt/ $\beta$-catenin pathway [19]. Kobayashi et al. first reported that sFRP2 has a function of promoting fibrosis. Their study found that the degree of myocardial fibrosis after myocardial infarction was reduced in sFRP2 knockout mice compared with normal mice [41]. Mastri et al. found that the administration of sFRP2 antibodies in hamsters with cardiomyopathy decreased apoptosis of cardiomyocytes, reduced myocardial fibrosis and improved cardiac function [42]. These findings support the proposal that sFRP2 can act as a profibrotic mediator [43]. Kobayashi and Mastri et al. proposed the following possible mechanism for sFRP2 promoting fibrosis. Procollagen processing of tolloid-like proteases exerts a rate-limiting effect during myocardial fibrosis, whereas sFRP2 enhances procollagen $\mathrm{C}$ protease activity in mammalian bone morphogenetic protein 1 (BMP1), resulting in the conversion of procollagen to collagen in the extracellular matrix, ultimately promoting collagen deposition and fibrosis [41, 42]. Similarly, Schuetze et al. revealed that, in cardiac fibroblasts, sFRP2 increases tissue non-specific alkaline phosphatase activity by activating the canonical Wnt signaling pathway, which promotes myocardial fibrosis and vascular calcification [44].

However, $\mathrm{He}$ et al. found that the degree of myocardial fibrosis was also relatively reduced in a myocardial infarction mouse model injected with recombinant sFRP2 molecules [45]. In addition, other research also demonstrated that $\mathrm{sFRP} 2$ can play a role in reducing myocardial fibrosis via regulating the BMP1 pathway [46]. Interestingly, these studies all noted that sFRP2 appears to affect cardiac fibrosis by affecting the BMP1 pathway, rather than through the canonical Wnt signaling pathway. Recently, Zhu et al. found that sFRP2 and procollagen C-proteinase enhancer 1 exert a synergistic effect on BMP1 in collagen formation. After silencing sFRP2 in vivo, Wnt signaling was inhibited, and collagen deposition and fibrosis were reduced [47].

How might we explain the apparently inconsistent role of sFRP2 in myocardial fibrosis in these studies? Mastri et al. speculated that high doses of sFRP2 can effectively inhibit the canonical Wnt signaling in the myocardium, while producing 
anti-fibrotic effects [42]. Similarly, the work of Alfaro et al. has revealed that low levels of sFRP2 $(<1$ $\mu \mathrm{g} / \mathrm{mL}$ ) promote procollagen $\mathrm{C}$ protease activity, which in turn promotes fibrosis, whereas high concentrations of sFRP2 ( $>6 \mu \mathrm{g} / \mathrm{mL})$ has the opposite effect [48]. Based on these studies, we propose that sFRP2 may play a similar two-way role in myocardial fibrosis (Figure 3A, B). A low concentration of sFRP2 can enhance the effects of Wnt pathway and promote myocardial fibrosis, while a high concentration of sFRP2 can antagonize the Wnt pathway and inhibit myocardial fibrosis. This bidirectional effect appears to be quite common during the fibrotic process in other organs. For example, Rajasekaran et al. observed that high-dose sFRP2 injection can inhibit Wnt signaling and reduce fibrosis in anal external sphincter injury in rabbits [49].

We do not yet completely understand the specific mechanisms of sFRP2 in fibrosis. Genetic differences in model systems, crosstalk between Wnt and other signaling pathways, and the bidirectional effects of sFRP2 on the Wnt signaling pathway highlight the complexity of the relationship between sFRP2 and cardiac fibrosis. It is possible that sFRP2 plays different roles at different stages of the development of myocardial fibrosis.

\section{Role of sFRP2 in angiogenesis}

Angiogenesis is a complex process which can be summarized as four steps: the induction of tip cells, sprout elongation, vascular branching and stabilization of the newly formed vascular network [50]. A large number of studies have proved that Wnt proteins play an important role in regulating the function of endothelial cells, tip cells, stalk cells during angiogensis via either canonical Wnt or non-caonical Wnt signaling pathway [51-53].
sFRP2 exerts pro-angiogenic effects through activation of non-canonical Wnt $/ \mathrm{Ca}^{2+}$ pathways. In mouse and chicken chorioallantoic membranes, sFRP2 was also found to induce angiogenesis [54]. It inhibits hypoxia-induced endothelial cell apoptosis, and induces endothelial angiogenesis by increasing endothelial cell migration. However, sFRP2 achieves the above functions through the Wnt/ $\mathrm{Ca}^{2+} / \mathrm{NFAT}$ (nuclear factor of activated $\mathrm{T}$ cells) pathway, without affecting the canonical Wnt pathway [55]. Similarly, sFRP2 has been shown to enhance angiogenesis in breast cancer through the above pathway [56]. A recent study revealed that frizzled-5 ( $F z d 5)$ also plays an important role during angiogenesis promoted by sFRP2. Endothelial cell tubes fail to form when $F z d 5$ is silenced in endothelial cells [57].

It is also generally understood that sFRP2 can exert an angiogenic effect through the above pathways in a wide variety of human tumors, including angiosarcoma, prostate cancer, renal cell carcinoma, lung cancer, and pancreatic cancer [58-60]. Crowley and coworkers reported that the addition of exogenous sFRP2 to adipose tissue promoted the expression of vascular endothelial growth factor mRNA, which suggested that sFRP2 may have a proangiogenic function in adipose tissue (Figure 3C) [61].

\section{Role of sFRP2 in cardiac hypertrophy}

Cardiac hypertrophy is an adaptive response of heart under many pathological states such as pressure overload and $\beta$-adrenergic stimuli [62]. Different signaling pathways and key molecules were involved in this process, including NFAT, phosphoinositide-3 kinase/protein kinase B (PI3K/AKT), extracellular regulated protein kinases (ERK), c-Jun N-terminal kinase (JNK), calcineurin and Wnt signaling pathways
A

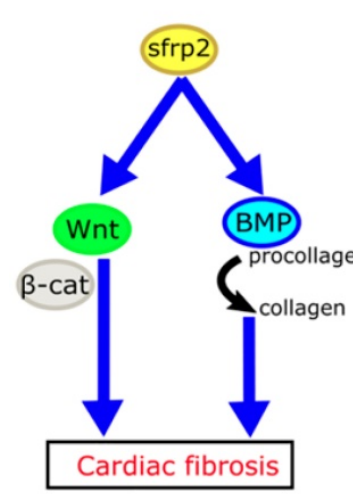

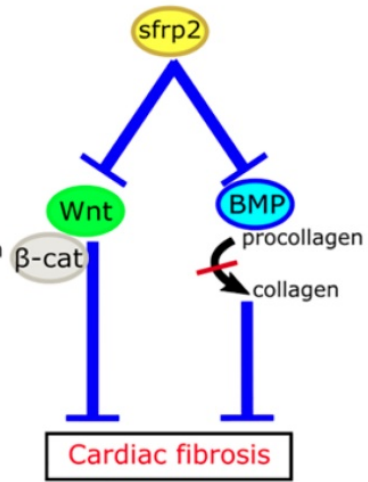

C

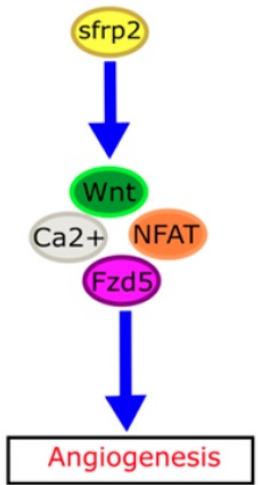

D

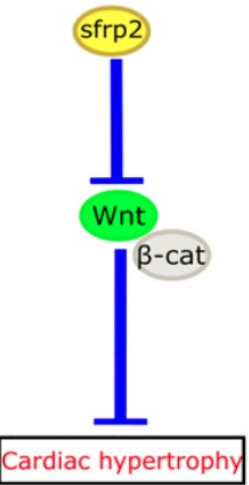

Figure 3: Role of sFRP2 in cardiovascular disease. (A) sFRP2 at low concentration activates the canonical Wnt/ $\beta$-catenin pathway and enhances procollagen $C$ protease activity in mammalian bone morphogenetic protein 1 (BMP1), eventually lead to cardiac fibrosis. (B) sFRP2 at high concentration inhibits the canonical Wnt/ 3 -catenin pathway and attenuates procollagen $C$ protease activity in mammalian BMP1, eventually leading to inhibition of cardiac fibrosis. (C) sFRP2 induces endothelial angiogenesis via the non-canonical Wnt/Ca ${ }^{2+} / \mathrm{NFAT} / F z d 5$ pathway. (D) sFRP2 protects the heart from hypertrophy by inhibiting the canonical Wnt/ $\beta$-catenin pathway. sFRP, secreted frizzle-related protein. 
$[62,63]$. He et al. reported that Wnt3a and Wnt5a were increased in hypertrophy mouse model induced by isoprenaline, which indicated that both canonical and non-canonical Wnt were involved in cardiac hypertrophy [64].

The HyperGEN study used linkage analysis in siblings with hypertension to determine that genetic variation in sFRP2 was associated with left ventricular hypertrophy [65]. Furthermore, a recent study has shown that sFRP2 can exert anti-atrophic effects on muscle cells by inhibiting transforming growth factor- $\beta 1$ in muscle cells [66]. Mohamed et al. have found that plasma membrane calcium ATPase (PMCA)4 acts as a key regulator of pathological cardiac hypertrophy by regulating sFRP2. Pharmacological blocking of PMCA4 can increase the expression of sFRP2 in cardiac fibroblasts, downregulate the canonical Wnt signaling pathway, and protect cardiomyocytes from pathological hypertrophy. These results suggest that sFRP2 may play a protective role in myocardial pathological hypertrophy [67] (Figure 3D).

\section{Role of sFRP2 in cardiac regeneration therapy}

The limited regenerative potential of cardiomyocytes has made cardiac regeneration therapy by stem cell transplantation a hotspot in cardiovascular disease research [68]. The therapeutic effect of stem cell transplantation depends on the survival and transplantation ability of these cells in the target organs. Due to the influence of local myocardial inflammation and fibrosis, the survival rate of stem cells after transplantation is low, significantly affecting the efficiency of cardiac transplantation [69]. To improve the effect of cell therapy, it will be necessary to enhance the viability of stem cells in the inflammatory and fibrotic environment created by ischemia-reperfusion injury.

Mesenchymal stem cells (MSCs) are believed to be a promising candidate stem cell for cardiac therapy $[70,71]$. Under hypoxic conditions, sFRP2 is thought to be a key regulator of the PI3K/AKT pathway in MSCs [48]. Blockage of the PI3K/Akt pathway or knockdown of sFRP2 with siRNA significantly increases the apoptosis of MSCs [72]. Gehmert et al. found that insulin-like growth factor-1 (IGF-1) plays an anti-apoptotic role by mediating sFRP2 activation in adipose tissue-derived stem cells; the release of sFRP2 is also dependent on the PI3K/Akt pathway [73]. Mirotsou et al. suggested that overexpression of Akt in MSCs can significantly increase their viability and survival rate after transplantation into the infarct myocardium, reduce the size of myocardial infarction, increase neovascular density, and ultimately improve cardiac function. These effects were dependent on the expression of sFRP2 as knocking out Sfrp 2 can abolish the aforementioned benefits [74]. After the injection of MSCs overexpressing sFRP2 around the infarcted myocardium, Alfaro et al. observed a downregulation of the canonical Wnt pathway [75]. Compared with the control group, the number of surviving MSCs was increased after myocardial injury, accompanied by increased vascular density, decreased infarct size, and significant improvement in cardiac function [75]. Pomduk et al. found that sFRP2 improved the viability of human MSCs (hMSCs) under oxidative stress, thereby improving the therapeutic effect of stem cell transplantation. However, sFRP2 did not affect the cell morphology, surface marker expression or differentiation potential of hMSCs [76]. This accumulation of evidence demonstrated that activation of the PI3K/Akt/sFRP2 pathway can protect the myocardium from apoptosis after MSCs transplantation [77, 78]. Our group also found that IGF-1 can promote the proliferation of MSCs and expression of C-X-C chemokine receptor type 4 (CXCR4), further promoting the migration of MSCs [79]. Moreover, we found that MSCs transplanted into rats with myocardial infarction can promote the expression of the anti-apoptotic gene $\mathrm{Bcl}-2$, inhibit cardiomyocyte apoptosis, and improve cardiac function [80].

Apart from MSCs, sFRP2 also has multiple effects on the properties of other types of stem cells. Aubert et al. found that sFRP2 can promote the differentiation of embryonic stem cells into neural progenitors by antagonizing the canonical Wnt signaling pathway [81], and subsequent studies have found that SFRP2 plays a key role in adipogenic and neuronal differentiation of dental tissue-derived MSCs [82]. Schmeckpeper et al. found that sFRP2 can enhance the differentiation of cardiac progenitor cells (CPCs) in vivo [83]. After myocardial ischemia-reperfusion injury, sFRP2 can double the number of new cardiomyocytes differentiated from CPCs, reduce infarct size, and improve cardiac function significantly. In this process, the canonical Wnt/ $\beta$-catenin signaling pathway was inhibited by sFRP2. At the same time, sFRP2 induces CPC cell cycle arrest and upregulates cardiac transcription factors by activating the non-canonical Wnt/JNK and Wnt/CaMKII pathways, which induce differentiation of CPCs into cardiomyocytes [83].

\section{Summary and future perspectives}

SFRP2 plays an important role in the process of heart development and a various cardiovascular pathophysiological condition. In myocardial fibrosis, sFRP2 may play a bidirectional effect depending on the different stage of disease and concentration. 
Subsequent studies had shown that sFRP2 can promote angiogenesis by activating the non-canonical Wnt/ $\mathrm{Ca}^{2+} / \mathrm{NFAT}$ pathway, while without affecting the canonical Wnt pathway. Furthermore, sFRP2 has been shown to downregulate the canonical Wnt signaling pathway, and protect cardiomyocytes from pathological hypertrophy. In cardiac regeneration therapy, sFRP2 can improve the survival of stem cells in the adverse environment, and enhance the therapeutic effects for myocardial infarction. Therefore, we believe that sFRP2 is a promising therapeutic target for multiple pathophysiological conditions of cardiovascular disease. However, the specific mechanisms of sFRP2 in these pathophysiological processes require further exploration. Interventions in the Wnt signaling pathway using modified sFRP2 expression may be a promising area of research for heart diseases.

\section{Abbreviations}

BMP1: bone morphogenetic protein 1; CPCs: cardiac progenitor cells; CRD: cysteine-rich domain; CXCR4: C-X-C chemokine receptor type 4; ERK: extracellular regulated protein kinases; ES: embryonic stem; Fz: frizzled; IGF-1: insulin-like growth factor-1; JNK: c-Jun N-terminal kinase; LEF/TCF: lymphocyte enhancement factor/T-cell factor; LRP: low-density lipoprotein receptor-related protein; MI: myocradial infarction; MSCs: mesenchymal stem cells; NFAT: nuclear factor of activated T cells; NTR: C-terminal netrin-like domain; PI3K/Akt: phosphoinositide-3 kinase/protein kinase B; PMCA: plasma membrane calcium ATPase; sFRP2: Secreted frizzled-related protein 2; wg: wingless.

\section{Acknowledgements}

The project was supported by the National Natural Science Foundation of China (No: 81600239), the Science and Technology Innovation Project from Foshan, Guangdong (FS0AA-KJ218-1301-0006) and the Clinical Research Startup Program of Shunde Hospital, Southern Medical University (CRSP2019001). We also acknowledge Michelle Kahmeyer-Gabbe, PhD, from Liwen Bianji, Edanz Editing China (www.liwenbianji.cn/ac), for editing the English text of a draft of this manuscript.

\section{Competing Interests}

The authors have declared that no competing interest exists.

\section{References}

1. Anitua E, Tejero R, Zalduendo MM, et al. Plasma rich in growth factors promotes bone tissue regeneration by stimulating proliferation, migration, and autocrine secretion in primary human osteoblasts. J PERIODONTOL. 2013; 84: 1180-90.
2. Sebio A, Kahn M, Lenz HJ. The potential of targeting Wnt/beta-catenin in colon cancer. Expert Opin Ther Targets. 2014; 18: 611-5.

3. Aubert J, Dunstan H, Chambers I, et al. Functional gene screening in embryonic stem cells implicates Wnt antagonism in neural differentiation. NAT BIOTECHNOL. 2002; 20: 1240-5.

4. Nusse R, van Ooyen A, Cox D, et al. Mode of proviral activation of a putative mammary oncogene (int-1) on mouse chromosome 15. NATURE. 1984; 307: 131-6.

5. Eisenberg LM, Eisenberg CA. Wnt signal transduction and the formation of the myocardium. DEV BIOL. 2006; 293: 305-15.

6. Barker N. The canonical Wnt/beta-catenin signalling pathway. Methods Mol Biol. 2008; 468: 5-15.

7. Logan CY, Nusse R. The Wnt signaling pathway in development and disease. Annu Rev Cell Dev Biol. 2004; 20: 781-810.

8. Valenta T, Hausmann G, Basler K. The many faces and functions of beta-catenin. EMBO J. 2012; 31: 2714-36.

9. Li X, Tamama K, Xie X, et al. Improving Cell Engraftment in Cardiac Stem Cell Therapy. STEM CELLS INT. 2016; 2016: 7168797.

10. Kawano $Y$, Kypta R. Secreted antagonists of the Wnt signalling pathway. J CELL SCI. 2003; 116: 2627-34.

11. Wang S, Krinks M, Lin K, et al. Frzb, a secreted protein expressed in the Spemann organizer, binds and inhibits Wnt-8. CELL. 1997; 88: 757-66.

12. Lin K, Wang S, Julius MA, et al. The cysteine-rich frizzled domain of Frzb-1 is required and sufficient for modulation of Wnt signaling. Proc Natl Acad Sci U S A. 1997; 94: 11196-200.

13. Bovolenta P, Esteve P, Ruiz JM, et al. Beyond Wnt inhibition: new functions of secreted Frizzled-related proteins in development and disease. J CELL SCI. 2008; 121: 737-46.

14. Yamamura S, Kawakami K, Hirata H, et al. Oncogenic functions of secreted Frizzled-related protein 2 in human renal cancer. MOL CANCER THER. 2010; 9: 1680-7.

15. Xiao X, Xiao $Y$, Wen $R$, et al. Promoting roles of the secreted frizzled-related protein 2 as a Wnt agonist in lung cancer cells. ONCOL REP. 2015; 34: 2259-66.

16. Kwack MH, Ahn JS, Jang JH, et al. SFRP2 augments Wnt/beta-catenin signalling in cultured dermal papilla cells. EXP DERMATOL. 2016; 25: 813-5.

17. Skah S, Nadjar J, Sirakov M, et al. The secreted Frizzled-Related Protein 2 modulates cell fate and the Wnt pathway in the murine intestinal epithelium. EXP CELL RES. 2015; 330: 56-65.

18. Esteve P, Sandonis A, Ibanez C, et al. Secreted frizzled-related proteins are required for $\mathrm{Wnt} /$ beta-catenin signalling activation in the vertebrate optic cup. DEVELOPMENT. 2011; 138: 4179-84.

19. Lin $\mathrm{H}$, Angeli $\mathrm{M}$, Chung KJ, et al. sFRP2 activates Wnt/beta-catenin signaling in cardiac fibroblasts: differential roles in cell growth, energy metabolism, and extracellular matrix remodeling. Am J Physiol Cell Physiol. 2016; 311: C710-9.

20. Olson EN. A decade of discoveries in cardiac biology. NAT MED 2004; 10 (5): 467-74.

21. Olson EN, Schneider MD. Sizing up the heart: development redux in disease. Genes Dev. 2003; 17: 1937-56.

22. Olson EN. Gene regulatory networks in the evolution and development of the heart. SCIENCE. 2006; 313: 1922-7.

23. Liu P, Wakamiya M, Shea MJ, et al. Requirement for Wnt3 in vertebrate axis formation. NAT GENET. 1999; 22: 361-5.

24. Huelsken J, Vogel R, Brinkmann V, et al. Requirement for beta-catenin in anterior-posterior axis formation in mice. J CELL BIOL. 2000; 148: 567-78.

25. Brault V, Moore R, Kutsch S, et al. Inactivation of the beta-catenin gene by Wnt1-Cre-mediated deletion results in dramatic brain malformation and failure of craniofacial development. DEVELOPMENT. 2001; 128: 1253-64.

26. Zamora M, Manner J, Ruiz-Lozano P. Epicardium-derived progenitor cells require beta-catenin for coronary artery formation. Proc Natl Acad Sci U S A. 2007; 104: 18109-14.

27. Marvin MJ, Di Rocco G, Gardiner A, et al. Inhibition of Wnt activity induces heart formation from posterior mesoderm. Genes Dev. 2001; 15: 316-27.

28. Naito AT, Shiojima I, Akazawa H, et al. Developmental stage-specific biphasic roles of Wnt/beta-catenin signaling in cardiomyogenesis and hematopoiesis. Proc Natl Acad Sci U S A. 2006; 103: $19812-7$.

29. Ueno S, Weidinger G, Osugi T, et al. Biphasic role for Wnt/beta-catenin signaling in cardiac specification in zebrafish and embryonic stem cells. Proc Natl Acad Sci U S A. 2007; 104: 9685-90.

30. Deb A, Davis BH, Guo J, et al. SFRP2 regulates cardiomyogenic differentiation by inhibiting a positive transcriptional autofeedback loop of Wnt3a. STEM CELLS. 2008; 26: 35-44.

31. Terry K, Magan H, Baranski M, et al. Sfrp-1 and sfrp-2 are expressed in overlapping and distinct domains during chick development. Mech Dev. 2000; 97: 177-82. 
32. Leimeister C, Bach A, Gessler M. Developmental expression patterns of mouse sFRP genes encoding members of the secreted frizzled related protein family. Mech Dev. 1998; 75: 29-42.

33. Satoh W, Matsuyama M, Takemura H, et al. Sfrp1, Sfrp2, and Sfrp5 regulate the Wnt/beta-catenin and the planar cell polarity pathways during early trunk formation in mouse. GENESIS. 2008; 46: 92-103.

34. Wittler L, Saborowski M, Kessel M. Expression of the chick Sizzled gene in progenitors of the cardiac outflow tract. GENE EXPR PATTERNS. 2008; 8: 471-6.

35. Ishizuya-Oka A, Kajita M, Hasebe T. Thyroid hormone-regulated Wnt5a/Ror2 signaling is essential for dedifferentiation of larval epithelial cells into adult stem cells in the Xenopus laevis intestine. PLOS ONE. 2014; 9: e107611.

36. Jeong $\mathrm{MH}, \mathrm{Kim} \mathrm{HJ}$, Pyun $\mathrm{JH}$, et al. Cdon deficiency causes cardiac remodeling through hyperactivation of WNT/beta-catenin signaling. Proc Natl Acad Sci U S A. 2017; 114: E1345-54.

37. Duan J, Gherghe C, Liu D, et al. Wnt1/betacatenin injury response activates the epicardium and cardiac fibroblasts to promote cardiac repair. EMBO J. 2012; 31: 429-42.

38. Paik DT, Rai M, Ryzhov S, et al. Wnt10b Gain-of-Function Improves Cardiac Repair by Arteriole Formation and Attenuation of Fibrosis. CIRC RES. 2015; 117: 804-16.

39. Duan J, Gherghe C, Liu D, et al. Wnt1/betacatenin injury response activates the epicardium and cardiac fibroblasts to promote cardiac repair. EMBO J. 2012; 31: 429-42.

40. Lal H, Ahmad F, Zhou J, et al. Cardiac fibroblast glycogen synthase kinase-3beta regulates ventricular remodeling and dysfunction in ischemic heart. CIRCULATION. 2014; 130: 419-30.

41. Kobayashi K, Luo M, Zhang Y, et al. Secreted Frizzled-related protein 2 is a procollagen $\mathrm{C}$ proteinase enhancer with a role in fibrosis associated with myocardial infarction. NAT CELL BIOL. 2009; 11: 46-55.

42. Mastri M, Shah Z, Hsieh K, et al. Secreted Frizzled-related protein 2 as a target in antifibrotic therapeutic intervention. Am J Physiol Cell Physiol. 2014; 306: C531-9.

43. Ostrom RS. A new molecular target for blunting organ fibrosis. Focus on "Secreted Frizzled-related protein 2 as a target in antifibrotic therapeutic intervention". Am J Physiol Cell Physiol. 2014; 306: C527-8.

44. Martin S, Lin H, Ejimadu C, et al. Tissue-nonspecific alkaline phosphatase as a target of sFRP2 in cardiac fibroblasts. Am J Physiol Cell Physiol. 2015; 309: C139-47.

45. He W, Zhang L, Ni A, et al. Exogenously administered secreted frizzled related protein 2 (Sfrp2) reduces fibrosis and improves cardiac function in a rat model of myocardial infarction. Proc Natl Acad Sci U S A. 2010; 107: 21110-5.

46. Lee HX, Ambrosio AL, Reversade B, et al. Embryonic dorsal-ventral signaling: secreted frizzled-related proteins as inhibitors of tolloid proteinases. CELL. 2006; 124: 147-59.

47. Zhu Q, Guo W, Zhang S, et al. Synergistic effect of PCPE1 and sFRP2 on the processing of procollagens via BMP1. FEBS LETT. 2019; 593: 119-27.

48. Alfaro MP, Vincent A, Saraswati S, et al. sFRP2 suppression of bone morphogenic protein (BMP) and Wnt signaling mediates mesenchymal stem cell (MSC) self-renewal promoting engraftment and myocardial repair. J BIOL CHEM. 2010; 285: 35645-53.

49. Rajasekaran MR, Kanoo S, Fu J, et al. Wnt-beta Catenin Signaling Pathway: A Major Player in the Injury Induced Fibrosis and Dysfunction of the External Anal Sphincter. Sci Rep. 2017; $7: 963$.

50. Sauteur L, Krudewig A, Herwig L, et al. Cdh5/VE-cadherin promotes endothelial cell interface elongation via cortical actin polymerization during angiogenic sprouting. CELL REP. 2014; 9: 504-13.

51. Zerlin M, Julius MA, Kitajewski J. Wnt/Frizzled signaling in angiogenesis. ANGIOGENESIS. 2008; 11: 63-9.

52. Vanhollebeke B, Stone OA, Bostaille $\mathrm{N}$, et al. Tip cell-specific requirement for an atypical Gpr124- and Reck-dependent Wnt/beta-catenin pathway during brain angiogenesis. ELIFE. 2015; 4

53. Phng LK, Potente M, Leslie JD, et al. Nrarp coordinates endothelial Notch and Wnt signaling to control vessel density in angiogenesis. DEV CELL. 2009; 16: 70-82.

54. Lin CT, Lin YT, Kuo TF. Investigation of mRNA expression for secreted frizzled-related protein 2 (sFRP2) in chick embryos. J Reprod Dev. 2007; 53: 801-10.

55. Courtwright A, Siamakpour-Reihani S, Arbiser JL, et al. Secreted frizzle-related protein 2 stimulates angiogenesis via a calcineurin/NFAT signaling pathway. CANCER RES. 2009; 69: 4621-8.

56. Siamakpour-Reihani S, Caster J, Bandhu ND, et al. The role of calcineurin/NFAT in SFRP2 induced angiogenesis--a rationale for breast cancer treatment with the calcineurin inhibitor tacrolimus. PLOS ONE. 2011; 6: e20412.

57. Peterson YK, Nasarre P, Bonilla IV, et al. Frizzled-5: a high affinity receptor for secreted frizzled-related protein-2 activation of nuclear factor of activated T-cells c3 signaling to promote angiogenesis. ANGIOGENESIS. 2017; 20: 615-28.

58. Fontenot E, Rossi E, Mumper R, et al. A novel monoclonal antibody to secreted frizzled-related protein 2 inhibits tumor growth. MOL CANCER THER. 2013; 12: 685-95.

59. Kim H, Yoo S, Zhou R, et al. Oncogenic role of SFRP2 in p53-mutant osteosarcoma development via autocrine and paracrine mechanism. Proc Natl Acad Sci U S A. 2018; 115: E11128-37.

60. Garcia D, Nasarre P, Bonilla IV, et al. Development of a Novel Humanized Monoclonal Antibody to Secreted Frizzled-Related Protein-2 That Inhibits Triple-Negative Breast Cancer and Angiosarcoma Growth In Vivo. ANN SURG ONCOL. 2019;26:4782-90

61. Crowley RK, O'Reilly MW, Bujalska IJ, et al. SFRP2 Is Associated with Increased Adiposity and VEGF Expression. PLOS ONE. 2016; 11: e163777.

62. van de Schans VA, Smits JF, Blankesteijn WM. The Wnt/frizzled pathway in cardiovascular development and disease: friend or foe? EUR J PHARMACOL. 2008; 585: 338-45.

63. Foldes G, Mioulane M, Wright JS, et al. Modulation of human embryonic stem cell-derived cardiomyocyte growth: a testbed for studying human cardiac hypertrophy? J MOL CELL CARDIOL. 2011; 50: 367-76.

64. He J, Cai Y, Luo LM, et al. Expression of Wnt and NCX1 and its correlation with cardiomyocyte apoptosis in mouse with myocardial hypertrophy. ASIAN PAC J TROP MED. 2015; 8: 930-6.

65. Arnett DK, Devereux RB, Rao DC, et al. Novel genetic variants contributing to left ventricular hypertrophy: the HyperGEN study. J HYPERTENS. 2009; 27: 1585-93.

66. Zhu X, Kny M, Schmidt F, et al. Secreted Frizzled-Related Protein 2 and Inflammation-Induced Skeletal Muscle Atrophy. CRIT CARE MED. 2017; 45: e169-83

67. Mohamed TM, Abou-Leisa R, Stafford N, et al. The plasma membrane calcium ATPase 4 signalling in cardiac fibroblasts mediates cardiomyocyte hypertrophy. NAT COMMUN. 2016; 7: 11074.

68. Blau HM, Daley GQ. Stem Cells in the Treatment of Disease. N Engl J Med. 2019; 380: 1748-60.

69. Toma C, Pittenger MF, Cahill KS, et al. Human mesenchymal stem cells differentiate to a cardiomyocyte phenotype in the adult murine heart. CIRCULATION. 2002; 105: 93-8.

70. Garikipati VN, Jadhav S, Pal L, et al. Mesenchymal stem cells from fetal heart attenuate myocardial injury after infarction: an in vivo serial pinhole gated SPECT-CT study in rats. PLOS ONE. 2014; 9: e100982.

71. Yang H, Li G, Han N, et al. Secreted frizzled-related protein 2 promotes the osteo/odontogenic differentiation and paracrine potentials of stem cells from apical papilla under inflammation and hypoxia conditions. Cell Prolif. 2019: e12694.

72. Zhou $\mathrm{H}$, Yang $\mathrm{J}$, Xin $\mathrm{T}$, et al. Exendin-4 protects adipose-derived mesenchymal stem cells from apoptosis induced by hydrogen peroxide through the PI3K/Akt-Sfrp2 pathways. Free Radic Biol Med. 2014; 77: 363-75.

73. Gehmert S, Sadat S, Song YH, et al. The anti-apoptotic effect of IGF-1 on tissue resident stem cells is mediated via PI3-kinase dependent secreted frizzled related protein 2 (Sfrp2) release. Biochem Biophys Res Commun. 2008; 371: 752-5.

74. Mirotsou M, Zhang Z, Deb A, et al. Secreted Frizzled Related Protein 2 (Sfrp2) Is the Key Akt-Mesenchymal Stem Cell-Released Paracrine Factor Mediating Myocardial Survival and Repair. P NATL ACAD SCI USA. 2007; 104: 1643-8.

75. Alfaro MP, Pagni M, Vincent A, et al. The Wnt modulator sFRP2 enhances mesenchymal stem cell engraftment, granulation tissue formation and myocardial repair. Proc Natl Acad Sci U S A. 2008; 105: 18366-71.

76. Pomduk K, Kheolamai P, U-Pratya $\mathrm{Y}$, et al. Enhanced human mesenchymal stem cell survival under oxidative stress by overexpression of secreted frizzled-related protein 2 gene. ANN HEMATOL. 2015; 94: 319-27.

77. Ni J, Liu X, Yin Y, et al. Exosomes Derived from TIMP2-Modified Human Umbilical Cord Mesenchymal Stem Cells Enhance the Repair Effect in Rat Model with Myocardial Infarction Possibly by the Akt/Sfrp2 Pathway. OXID MED CELL LONGEV. 2019; 2019: 1958941.

78. Yu G, Wang J, Lin X, et al. Demethylation of SFRP2 by histone demethylase KDM2A regulated osteo-/dentinogenic differentiation of stem cells of the apical papilla. Cell Prolif. 2016; 49: 330-40.

79. Huang YL, Qiu RF, Mai WY, et al. Effects of insulin-like growth factor-1 on the properties of mesenchymal stem cells in vitro. J Zhejiang Univ Sci B. $2012 ; 13: 20-8$

80. Huang $\mathrm{YL}, \mathrm{Kuang} \mathrm{J}, \mathrm{Hu} \mathrm{YZ}$, et al. Bone marrow stromal cell transplantation combined with angiotensin-converting enzyme inhibitor treatment in rat with acute myocardial infarction and the role of insulin-like growth factor-1. CYTOTHERAPY. 2012; 14: 563-9. 
81. Aubert J, Dunstan H, Chambers I, et al. Functional gene screening in embryonic stem cells implicates Wnt antagonism in neural differentiation. NAT BIOTECHNOL. 2002; 20: 1240-5.

82. Lin $\mathrm{X}$, Dong R, Diao S, et al. SFRP2 enhanced the adipogenic and neuronal differentiation potentials of stem cells from apical papilla. CELL BIOL INT. 2017; 41: 534-43.

83. Schmeckpeper J, Verma A, Yin L, et al. Inhibition of Wnt6 by Sfrp2 regulates adult cardiac progenitor cell differentiation by differential modulation of Wnt pathways. J MOL CELL CARDIOL. 2015; 85: 215-25. 